\title{
Proposed standard measurement techniques for the technical specification of biofeedback devices
}

\author{
CHARLES G. BURGAR and JOHN D. RUGH \\ Departments of Psychiatry and Restorative Dentistry, University of Texas Health Science Center
}

San Antonio, Texas 78284

\begin{abstract}
Standardized test procedures are proposed that may be used by instrument manufacturers, investigators, and testing agencies to specify the functional characteristics of biofeedback devices. The test procedures are intended for EMG, EEG, and EKG feedback devices. Included are procedures for the specification of electrode characteristics, input impedance, noise, common-mode rejection ratio (CMRR), filter characteristics, time constant, battery life, and characteristics of the feedback signal. A variety of novel test procedures are currently being used to specify biofeedback instrument functional characteristics. Adoption of common test procedures will improve comparability of equipment, technical specification sheets, and apparatus technical descriptions in research reports.
\end{abstract}

Although the clinical application of biofeedback is yet experimental, a large market has developed for biofeedback devices designed for clinical applications. There are currently 54 small companies marketing over 170 different biofeedback devices. Most of these devices are sold to health professionals for use in research or for the diagnosis and treatment of stress-related psychophysiological disorders. Factors limiting the use of these devices are the lack of standardization of instrument technical characteristics and of the procedures used to measure them.

The biofeedback devices currently available have been found to vary widely in important functional characteristics. In a comparative study of 11 commercial EMG instruments, Rugh and Schwitzgebel (1977) reported that input impedance varied from $13 \mathrm{kohm}$ to more than 1 megohm. Filter bandwidths varied from 55 to $2,600 \mathrm{~Hz}$. In an earlier comparative study (Schwitzgebel \& Rugh, 1975) of 13 alpha brain-wave feedback devices, input impedance was found to vary from $500 \mathrm{ohm}$ to $1 \mathrm{megohm}$ and the alpha-filter center frequency varied from 7.8 to $14 \mathrm{~Hz}$. Bandwidth varied from .9 to $16 \mathrm{~Hz}$. This wide variability in instruments makes it difficult, if not impossible, to compare the work from different laboratories or clinics. The results of these studies clearly indicate the need for development of standards.

At this time, technical standards cannot be suggested for most instrument characteristics, since little research has been done to determine those that are ideal. There is general agreement on a few instrument characteristics, such as input impedance and commonmode rejection. But optimum values for many other

Requests for reprints should be directed to John D. Rugh, Department of Restorative Dentistry, Dental School, University of Texas Health Science Center, San Antonio, Texas 78284. important functional characteristics, such as bandpass, nature of the feedback signal, and time constant, have not been experimentally determined. An attempt at this time to standardize biofeedback instrumentation characteristics would be premature and would likely stifle the innovative efforts of manufacturers and researchers.

The situation can be improved, however, by standardizing manufacturers' test and calibration procedures. Currently, each manufacturer selects from a wide variety of test procedures to specify the technical characteristics of their instruments. Different test procedures often provide different results. This does not allow the user to make meaningful comparisons between instruments. Occasionally, unorthodox test procedures are used that prohibit meaningful technical descriptions of the devices in research reports. Finally, without comparable performance data, the consumer cannot compare the cost effectiveness of different devices. Battery life, for example, has been found to vary from $15 \mathrm{~h}$ to over $500 \mathrm{~h}$. Unfortunately, manufacturers' specification sheets often do not reflect such important differences.

It is the purpose of this paper to propose a set of test and calibration procedures for use in specifying the functional characteristics of clinical EEG, EMG, and EKG biofeedback devices. As such, it presents a set of procedures that may be amended as required by future technical developments and research. Where possible, the authors have attempted to use standard nomenclature and procedures common to other biomedical instrumentation.

\section{PRELIMINARY CONSIDERATIONS AND TEST INSTRUMENTS}

(1) All tests should be performed on devices as they are intended to be used. If performance is degraded 
by accessory equipment, these conditions must be simulated.

(2) Tests are made with battery-operated test instruments and/or in electrically shielded rooms to facilitate accurate measurements and reduce $60-\mathrm{Hz}$ interference.

(3) Test instrument calibration must be National Bureau of Standards traceable and made within the calibration interval recommended by the manufacturer.

(4) All performance specifications should be provided in minimum values so that all devices manufactured will meet the listed specifications. Where variability is expected because of component tolerance, an attempt should be made to indicate the expected variability or tolerance. System gain, for example, should be given as $80 \mathrm{~dB}( \pm 1 \mathrm{~dB})$.

(5) Amplifier specifications, such as input impedance, common-mode rejection, and filter characteristics, should be made through the electrode cables normally supplied with the device.

(6) When recommended test procedures cannot be applied because of unusual design techniques, the manufacturer shall so note and supply a detailed description of the alternative test procedure.

(7) Sine-wave harmonic distortion of signal generators employed in these tests must not exceed .1\%.

(8) Accuracy of amplitude and frequency measurements must be $1 \%$ or better. Alternating current (ac) amplitudes shall be measured with an ac-coupled rootmean-square (RMS) responding device rated for $1 \%$ or better accuracy over the frequency range encountered.

\section{PROPOSED TEST PROCEDURES}

\section{Electrodes}

The nature of the electrode greatly influences the system noise, signal amplitude, signal variability over time, and the skin-electrode impedance (Geddes, 1972). It would be desirable to have published specifications regarding electrode polarization, typical skin-electrode impedance, and noise. Since these characteristics are highly dependent upon the user's application and procedures, standard test procedures have not been developed. Specification sheets should, however, include a description of: (1) the type of electrode material $(\mathrm{Ag} / \mathrm{AgCl}$, stainless steel, etc.), (2) the electrode surface area, (3) the basic design (recessed vs. direct skin contact, sponge, disposable, or resuable, etc.), (4) the length of the electrode cable, (5) method of attachment to the subject, and (6) cost per application of expendable items.

\section{0-Hz Suppression}

The ability of a bioamplifier to reject $60-\mathrm{Hz}$ noise is dependent upon several instrument design and performance characteristics. Some commercial biofeedback units have $60-\mathrm{Hz}$ notch filters, most have good commonmode rejection characteristics, and many have bandpass filters that reduce the instrument gain at $60 \mathrm{~Hz}$. Each of these contributes to the device's ability to reject
$60-\mathrm{Hz}$ noise and must be specified independently. It is useful to have one collective measure that provides an indication of the instrument's ability to reject $60-\mathrm{Hz}$ noise. Such a measure is provided below.

Technique. The $60-\mathrm{Hz}$ suppression figure may be calculated by the following formula:

$$
20 \text { Log } \frac{\text { Differential Gain (midband) }}{\text { Common-Mode Gain }(60 \mathrm{~Hz})} \text {. }
$$

These gain measurements are made as described later in the Technique section for common-mode rejection ratio and in Figures 2 and 3 . It is recommended that the "midband" be defined as the geometric mean rather than the algebraic mean, as most filters are not symmetrical. The geometric mean may be calculated as follows:

$$
F_{G M}=\sqrt{F_{1} \times F_{2}} .
$$

$F_{1}$ represents the lower $-3-d B$ point, and $F_{2}$ represents the upper $-3-\mathrm{dB}$ point.

\section{Battery Life}

The cost effectiveness of a device cannot be adequately estimated without knowledge of the type of batteries used and the expected battery life. When rechargeable batteries are employed, it is desirable to know both the charge and discharge time.

Technique. Battery life is tested under worst-case conditions, that is, continuous use with an input signal and with the device in the operating mode that draws the most current. Ambient temperature during this measurement should be between $70^{\circ} \mathrm{F}$ and $80^{\circ} \mathrm{F}$. The batteries should be considered dead when a $10 \%$ degradation in any of the performance criteria is observed. This may be, but is not limited to, a reduction in gain, a loss of audio volume, distortion of the amplified signal, or a reduction in the frequency of the voltage-controlled oscillator used for feedback. The battery employed in the test must be specified (e.g., Everready 522 Alkaline 9. $\mathrm{V}$ batteries).

\section{Input Impedance}

The accuracy, common-mode rejection, and $60-\mathrm{Hz}$ suppression characteristics are dependent in part on the device's input impedance (Pacela, 1967). Accurately measuring this parameter is probably more difficult than any of the other tests listed, but the information obtained is among the most important. Many biofeedback instrument manufacturers rely on the semiconductor data sheet for the device used in the input stage for this information. In general, this results in a figure much higher than the actual impedance due to the loading effects of circuit capacitance, input voltage limiting devices, RF bypassing components, and, as the authors have observed, significant capacitance in the electrode cables. The following measurement technique provides a compromise that reflects the 
performance of the system under normal usage and includes the error sources mentioned above. Since the common-mode input impedance is usually much higher than the differential-mode impedance, only the latter is determined here.

Technique. The differential input impedance is specified as a minimum dc resistance in parallel with a maximum capacitance (e.g., 10 megohm $\| 310$ picoF). The resistance value may be calculated mathematically, using the input resistance figure provided by the manufacturer for the input stage device along with the values of any serics or parallel resistance added by the circuitry of the biofeedback instrument. Alternatively, for input stages employing dc coupling and having low gain, this figure may be measured by applying a known differential dc voltage (less than that which would cause saturation of this stage), measuring the resulting input current flow with a picoammeter (HewlettPackard 419A or equivalent), and taking the ratio of these two quantities. The latter method is limited to those amplifiers having input resistance of approximately 10 megohm or less.

The input capacitance is measured by removing the input amplifier device(s) from the circuit and connecting the active electrode leads to a capacitance meter having an accuracy of $5 \%$ or better (Hewlett-Packard 4332A or equivalent). The authors have found the major contribution to input capacitance to come from the electrode cables and input RF bypass components, with a negligible error $(<1 \%)$ resulting from the removal of the input amplifier device.

This test should be performed with a sufficiently large sample of electrode cables and feedback instruments to insure that those figures claimed represent the minimum impedance expected, including allowance for normal manufacturing tolerances. From the resistance and capacitance values, the midband input impedance (ac) should be calculated and specified for the geometric mean frequency of the widest bandpass of the instrument using the formula:

$$
Z_{I N}=\frac{R_{I N} X_{C}}{\sqrt{R_{I N}{ }^{2}+X_{C}{ }^{2}}} .
$$

\section{Noise}

The ability to detect the extremely small potential differences found at the surface of the skin while monitoring relaxed muscles, high-frequency brain-wave activity, and so on, is limited by the intrinsic noise of the measuring system. While noise figure is a common measurement for comparing the performance of amplifiers, its minimum at a nonzero source resistance can be misinterpreted as indicating some "best" value of source impedance, other than zero (Graeme, 1973). We recommend the use of an equivalent input noise specified with a standardized impedance connected to the amplifier input. This parameter then reflects the noise contributions from both input noise voltage and current sources.

Technique. The device to be tested is connected to all accessories normally used that may result in an increase in input equivalent noise voltage $\left(e_{n i t}\right)$.

A standard test load is connected from each active input of the device to the reference input through the patient electrode cable (see Figure 1). An ac-coupled RMS responding meter having an accuracy of $1 \%$ or better and a frequency response greater than that of the amplifier is then used to measure the noise voltage level at the output of the filter stages, if any, but before the stage where detection of the signal is accomplished. This RMS voltage level is divided by the small signal gain at this point in the circuit to yield the equivalent input noise voltage $\left(e_{n i t}\right)$ in microvolts RMS.

This test is to be made and reported for each available filter bandwidth setting. For instruments having a large number of bandpass combinations available, $e_{n i t}$ may be specified for only the standard or recommended settings found in the operating manual and, additionally, for the widest bandpass available.

\section{Common-Mode Rejection Ratio (CMRR)}

Since most biofeedback training takes place without the benefit of electromagnetic or electrostatic shielding of the subject, the instrument used must be capable of detecting microvolt-level signals generated within the subject in the presence of interfering signals having magnitudes as high as several volts, induced in the

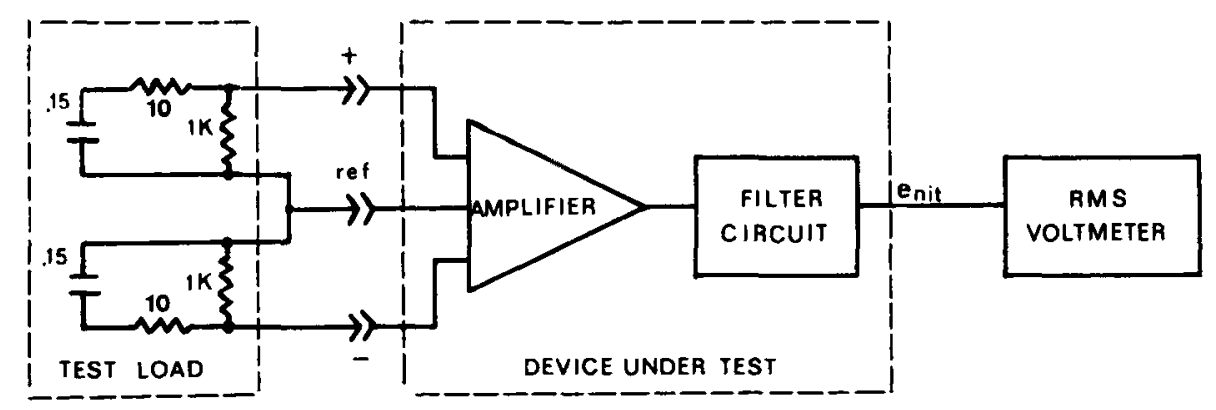

Figure 1. Test circuit for measurement of input equivalent noise voltage $\left(e_{\text {nit }}\right)$. Capacitance is shown in microfarads. Resistors are metal film type. 


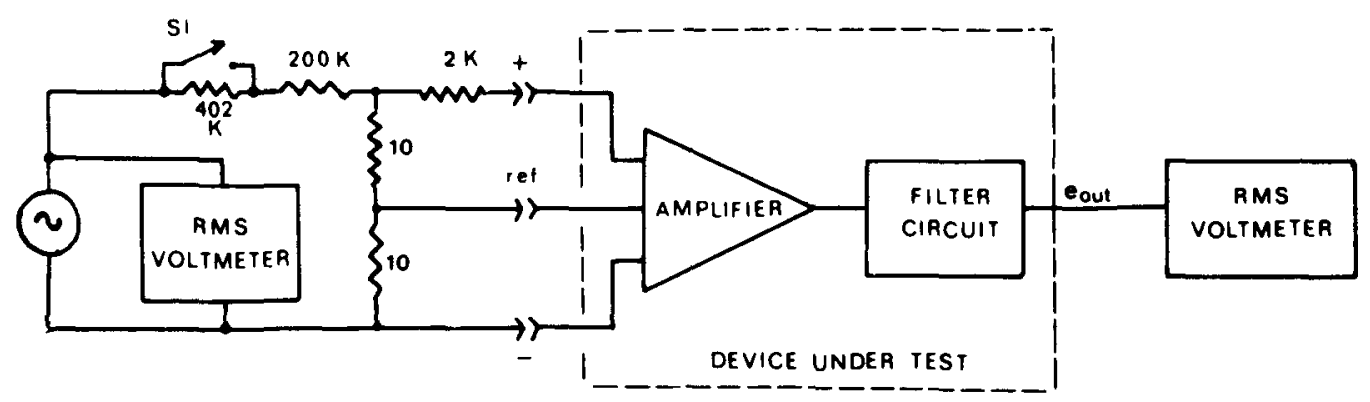

Figure 2. Test circuit for differential gain measurement including a 10,000:1 attenuator. All resistors are 1\% metal film. The switch (S1) is a normally closed type. It is used to introduce a step change for time constant measurements. The sine-wave oscillator output impedance is $\$ 600 \mathrm{megohm}$. The generator output divided by the attenuation factor equals $\mathrm{e}_{\mathrm{in}}$.

measuring system by external sources. The ability of a differential input amplifier to reject such artifact, as well as those interfering signals generated within the subject at sites other than that being monitored, is measured by the CMRR of the amplifier. This parameter has been defined as the ratio of the differential gain to the common-mode gain (Gans, 1969).

Since the dominant artifact encountered in monitoring physiological parameters is coupled from the $60-\mathrm{Hz}$ power lines, many manufacturers of biofeedback instruments include $60-\mathrm{Hz}$ notch filters in their devices to provide additional attenuation of artifact signals from this source. A common-mode rejection measurement made at $60 \mathrm{~Hz}$ does not, therefore, accurately reflect the ability of the feedback instrument to reject unwanted signals with frequencies other than $60 \mathrm{~Hz}$. Also, source impedance imbalance results in common-mode signals being converted to differential-mode signals. It is, therefore, recommended that the common-mode rejection ratio be measured both at $60 \mathrm{~Hz}$ and at the geometric mean of the amplifier passband with a $2-\mathrm{kohm}$ source imbalance.

Technique. Using the testing configuration of Figure 2, the differential-mode gain is measured as the ratio of $e_{\text {out }}$ to $e_{\text {in }}$ at $60 \mathrm{~Hz}$ and at the geometric mean of the widest bandpass available on the instrument.

Utilizing the test configuration of Figure 3 , the common-mode gain is also measured as the ratio of $e_{o u t}$ to $e_{i n}$ at $60 \mathrm{~Hz}$ and at the geometric mean frequency. The common-mode rejection ratios, expressed in decibels, are calculated as:

CMRR (60 Hz, 2K Imbalance)

$$
=\frac{\text { Differential Gain }(60 \mathrm{~Hz})}{\text { Common-Mode Gain }(60 \mathrm{~Hz})},
$$

CMRR (Midband, 2K Imbalance)

$$
=\frac{\text { Differential Gain (Midband) }}{\text { Common-Mode Gain (Midband) }} .
$$

\section{Filter Response}

Most biofeedback instruments utilize one or more filter stages to limit the response of the instrument to those frequencies present in the signal of interest. Although marketed to perform the same function, instruments produced by different manufacturers have been found to vary widely in their frequency response characteristics (Rugh \& Schwitzgebel, 1977). To facilitate the evaluation of individual biofeedback instruments and to allow comparison between instruments of different manufacturers, curvilinear plots of the output response of the biofeedback unit vs. frequency should be provided in all sales literature in

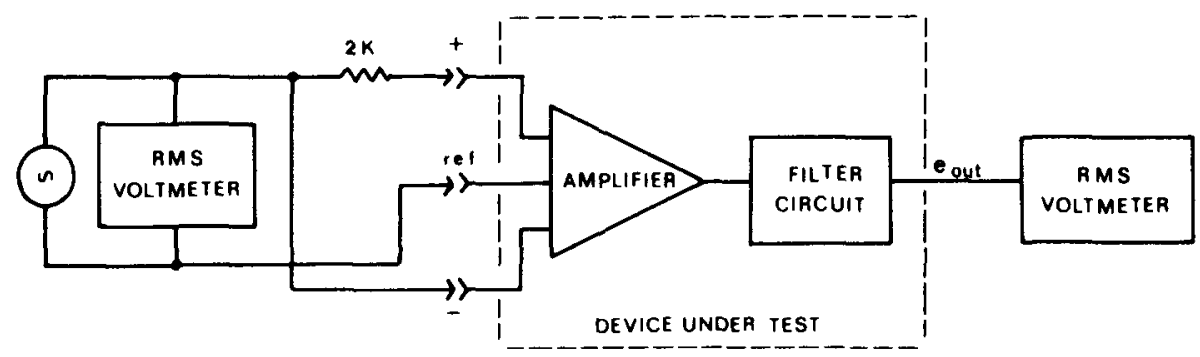

Figure 3. Test circuit for common-mode gain measurement. The resistor is $1 \%$ metal film type. The sine-wave oscillator output impedance is $6600 \mathrm{ohms}$. The gain or sensitivity of the device under test should be set at midrange. 
which technical characteristics are cited. A separate curve should be provided for each filter bandpass available unless, as is the case with some EEG feedback devices, a large number of upper and lower filter settings are available. In such case, the graphs would be given for each bandpass normally used or recommended in the instruction manual.

Technique. When testing EMG and EEG equipment, a linear representation of the detector output is plotted on the vertical axis against the $\log$ of input frequency on the horizontal axis. The filter response for EKG instruments is made by detecting the output of the filter stages with a true RMS responding device and plotting the magnitude on the horizontal axis. If an EMG or EEG feedback device lacks a linear output signal, the graph of the filter response is made as for the EKG test given above. All graphs are made to cover the frequency range from one decade below to one decade above the half-power points of the filter under test. If the instrument under test has more than one gain setting, the filter curves are made for the range closest to the middle of the dynamic range of the instrument.

The following measurements are also made and reported in any listing of the technical specifications for a device. If a 60-cycle notch filter is included, the 60-cycle notch depth, expressed in decibels below the filter's response at the geometric mean frequency, is measured and reported. The bandpass of the filter, in hert $z$, is measured as the difference between the upper and lower cutoff $(-3-\mathrm{dB})$ points of the filter. Rolloff, in decibels per octave, is measured as the average slope of the filter response curve starting at the $-3-\mathrm{dB}$ points and extending for two octaves outside the bandpass. If the two values are different, the rolloff is reported for both the high- and low-pass filter skirts. Since most filter designs yield frequency response characteristics that are not symmetrical about the algebraic mean of the band, the center frequency is measured and reported as the geometric mean frequency, described previously.

\section{Audio Output}

The minimum audio output power and the impedance into which this power is delivered are useful parameters for evaluating biofeedback instruments having an audio feedback mode. Such information might be used when selecting devices to be used for demonstrations before large groups. Knowledge of the intended load impedance allows the proper type headphone or external speaker to be purchased separately, if desired.

Technique. For those devices having a steady or pulsating tone output, the RMS value of the output power is determined by measuring the RMS voltage delivered into a resistive load that has a resistance equal to the recommended load impedance. The power is determined mathematically as the ratio of the square of the output voltage divided by the load resistance.
Instruments whose audio output consists of a series of clicks are rated as to their peak power output, determined by taking the ratio of the square of the peak output voltage divided by the load resistance. In either case, the measurement should be made with the volume control at its maximum setting and with all auxiliary equipment connected and operating. The term peak or RMS is used to indicate which test method was used.

\section{Operating Temperature Range}

A range of temperatures over which the technical specifications are guaranteed should be determined and included with the other technical data on the equipment-specification sheets.

Technique. Ideally, a chamber in which the temperature and humidity can be controlled is used to vary the operating conditions so that any sensitivity to ambient temperature change is detected. Alternate techniques may be used to vary the ambient temperature, but the instrument should be tested for proper operation over a temperature range of at least $60^{\circ}$ to $90^{\circ} \mathrm{F}$. All specifications claimed for a device should be valid over at least this temperature range.

\section{Signal Processing}

To enable users to compare results obtained with biofeedback instruments produced by different manufacturers, information as to the signal-detection method and the units in which the display is calibrated (RMS, peak to peak, average, etc.) should be given.

Technique. A verbal description of the input-output (I/O) relationship between an input level or change and the resulting feedback response should be provided. Such a description would contain information about the characteristics of the feedback signal, such as whether the output responds linearly or nonlinearly to the input. For derivative feedback modalities, the rate of change of the input necessary to produce a given output should be specified. Graphs showing the I/O relationship for each mode of feedback and representing the range of sensitivities available should accompany the verbal description.

\section{Accuracy}

While a relative change of a physiological parameter is usually the goal of biofeedback training, the ability to accurately quantify such changes, as well as the initial and final values, is necessary when making comparisons between subjects, between sessions, or between biofeedback training facilities. Along with other factors that are equally important, the overall accuracy of instrument readings must be known in order to determine the amount of variability to expect between instruments of the same manufacturer and model. Due to the differences in filter characteristics and detection methods, comparison of signal amplitudes cannot be made between instruments of different manufacturers. 
Technique. Those feedback instruments responding to the RMS, peak-to-peak, or average value of the amplitude of the input signal are calibrated using a sine wave with less than $.1 \%$ harmonic distortion and having a frequency equal to that of the geometric mean of the passband. The tolerance associated with such calibration is given as a total percentage and includes the sum of the tolerances of the signal-generating and measuring equipment, as well as manufacturing tolerances.

\section{Auxiliary Instrumentation Output Jacks}

To facilitate the interfacing of auxiliary equipment to biofeedback instruments, certain information is needed. Many researchers use auxiliary recording and display devices, and a growing number of facilities are in the process of interfacing small computer systems to their biofeedback instruments to automate the collection and the scoring of data. Safe and accurate interfacing depends on the availability of the following information.

Technique. The output waveform, sensitivity, range, and impedance are given for each auxiliary output jack. If the output is optically isolated, the breakdown voltage and the leakage at $60 \mathrm{~Hz}$ are also indicated.

\section{Time Constant}

The speed with which the feedback signal responds to a change in input level depends on the time constant of the instrument's circuitry. The input signal change may consist of differences in amplitude and/or frequency. Although research has yet to be done to determine optimum response time, many users feel that the faster the reinforcement, the sooner control will be learned.

Technique. Instruments that feed back information on the amplitude of EMG activity are subjected to a step increase from $25 \%$ to $75 \%$ of the full-scale value of the range that is closest to the middle of the instrument's total range. This is accomplished by using the test circuit of Figure 2, adjusting the signal generator for an output equal to $25 \%$ of full scale with Switch 1 (S1) open, then closing the switch, which causes the attenuator output to increase to $75 \%$ of full scale. The time required for the output of the feedback instrument to achieve $63 \%$ of the change to its final value gives the time constant. Devices with selectable time constants are tested at the minimum and maximum values available.

Heart rate feedback instruments are subjected to a step pulse rate change from $25 \%$ to $50 \%$ of the instrument's dynamic range, and the time constant is taken as the time required for the output display to indicate at least $63 \%$ of the total change.

For EEG biofeedback instruments, the time constant is specified for both frequency and amplitude input changes. The amplitude response time constant is measured by determining the number of cycles of input signal that occur before feedback is initiated after the input, centered at the geometric mean of the selected bandpass, is stepped from $50 \%$ to $150 \%$ of threshold, using the circuit of Figure 2. The frequency response time constant is measured as the number of cycles of input signal that occur before feedback is initiated following $a$ decade step change in input frequency from below the bandpass to the geometric mean of the bandpass. During this test, the input signal amplitude is maintained at $150 \%$ of threshold. The frequency band used in making this test is specified.

Time constant measurements are most conveniently made by observing the input and output signals on a storage oscilloscope or, where appropriate, on a chart recorder.

\section{Maximum Fault Current Through Subject $\left(\mathbf{I}_{\mathrm{F}}\right)$}

Most feedback instruments incorporate some means of limiting the current through the subject should a component failure occur. Knowing the maximum value of this current not only allows the user to assess the safety aspects of the device, but also to choose those instruments that would cause the least discomfort to a subject in the event of a malfunction.

Technique. The steady-state fault current through a resistance of a kohm connected from each active electrode to the reference electrode is determined by inspection of the schematic diagram of the input circuit for the worst-case failure possible of active components and/or polarized capacitors. For the purpose of this test, resistors, nonpolarized capacitors having a voltage rating at least $50 \%$ greater than the maximum potential difference between power supplies, sockets, switches, wiring, and printed circuit conductors are not considered to be subject to failure. Each active (semiconductor) device is evaluated using internal circuitry information provided by its manufacturer, and the failure mode is determined that would result in the largest current flow through the subject. A similar analysis is performed for each passive component, other than those exempted above. Only one component failure is simulated at a time, unless the failure of one component could reasonably be expected to lead to the failure of others. The current $I_{F}$ is taken as the largest of the currents determined from the above analysis.

\section{DISCUSSION}

The use of standard measurement techniques for specifying instrument characteristics as proposed here must be differentiated from the development of instrument technical standards. Technical standards require that instruments meet specific operational and technical criteria, such as 1 -megohm input impedance, .5 -sec time constant, and $100-\mathrm{Hz}$ bandpass. The development of commercial biofeedback instrumentation is still at an experimental stage, and ideal instrument characteristics 
have not been identified. It is thus too early to specify many instrument technical characteristics. The measurement standards proposed here do not set requirements for operational characteristics; rather, they simply allow different device characteristics to be measured using standardized techniques so that they are comparable.

Although the above measurement techniques cover a wide range of important operational characteristics, they are not comprehensive. Lacking, for example, is a procedure that allows comparison of meter readings of different EMG devices. Most EMG devices are currently calibrated with a sine wave at the center of the device's bandpass. The bandwidth and center frequency of different devices vary, and since agreement has not been reached on the frequency spectrum of the EMG signal, a standard test signal cannot be specified at this time. Without an agreed-upon test signal, it is futile to attempt development of a standardized test procedure. Future research needs to be directed at clarifying the frequencypower spectrum of low-level EMG signals. Based upon this research, a standard test signal and test procedure may then be developed.

The proposed test procedures have been verified on a wide variety of commercial biofeedback devices. Tested devices varied in front-end design, filter methods, and signal processing. The proposed test procedures were applicable to each instrument. There was some difficulty testing instruments with "potted" front-end circuitry. Measurement of input capacitance requires that the input semiconductor devices be removed. But removal is impossible in instruments whose circuits have been encapsulated in plastic. This is not likely to be a limiting factor, as fewer manufacturers are currently potting circuit boards. Also, measurements can be made prior to potting.

The Food and Drug Administration, working under the new medical device laws, has classified biofeedback devices in Category II, which requires, among other things, that the devices be regulated by standards. Such standards have not been developed but will likely include specifications for safety, performance, and therapeutic efficacy. The standards and regulations will ideally be developed by responsible professional societies, manufacturers' organizations, and representatives of public interest groups. If these societies and organizations do not accept this responsibility, the federal government undoubtedly will. The Biofeedback Society of America and the American Association of Biofeedback Manufacturers have been working since 1976 with the second author to establish safety standards for commercial biofeedback devices. The Association for the Advancement of Medical Instrumentation Safe Current Limits Standard (SCLP10-75) was accepted by both organizations in March of 1978. The standards proposed here may serve as guidelines for the next phase of standards acceptance, which will include a set of common measurement techniques for specifying device technical characteristics.

\section{REFERENCES}

GaNs, F. Common-mode rejection ratio: What the specification sheet doesn't say. Electronics, June 23, 1969, pp. 116-119.

GEDDEs, L. A. Electrodes and the measurement of bioelectric events. New York: Wiley, 1972.

GRAEME, J. G. Applications of operational amplifiers-Third generation techniques. New York: McGraw-Hill, 1973.

Pacela, A. F. Collecting the body's signals. Electronics, July 10,1967 , pp. 103-112.

Rugh, J. D., \& Schwitzgebel, R. L. Variability in commercial electromyographic biofeedback devices. Behavior Research Methods \& Instrumentation, 1977, 9, 281-285.

Schwitzgebel, R. L., \& Rugh, J. D. Of bread, circuses and alpha machines. American Psychologist, 1975, 30, 363-370. 